\title{
Antihepatitis B Surface Antigen and Hepatitis C Antibodies among Pregnant Women in an Urban Area of Mwanza City, Tanzania
}

\author{
Elieza Chibwe, ${ }^{1}$ Vitus Silago, ${ }^{2}$ Edwin Kajoro, ${ }^{2}$ Muhsin Juma, ${ }^{2}$ Emmanuel Mkumbo, ${ }^{2}$ \\ Caroline A. Minja, ${ }^{3}$ Fridolin Mujuni, ${ }^{1}$ Stephen E. Mshana $\mathbb{D}^{2},{ }^{2}$ and Mariam M. Mirambo $\mathbb{D}^{2}$ \\ ${ }^{1}$ Department of Obstetrics and Gynecology, Weill Bugando School of Medicine, Catholic University of Health and Allied Sciences, \\ P.O. Box 1464, Mwanza, Tanzania \\ ${ }^{2}$ Department of Microbiology and Immunology, Weill Bugando School of Medicine, Catholic University of Health and Allied Sciences, \\ P.O. Box 1464, Mwanza, Tanzania \\ ${ }^{3}$ Department of Biochemistry and Molecular Biology, Weill Bugando School of Medicine, Catholic University of Health and \\ Allied Sciences, P.O. Box 1464, Mwanza, Tanzania
}

Correspondence should be addressed to Mariam M. Mirambo; mmmirambo@gmail.com

Received 10 December 2018; Revised 14 May 2019; Accepted 30 May 2019; Published 18 June 2019

Academic Editor: Luca Marozio

Copyright (C) 2019 Elieza Chibwe et al. This is an open access article distributed under the Creative Commons Attribution License, which permits unrestricted use, distribution, and reproduction in any medium, provided the original work is properly cited.

Background. Hepatitis B and hepatitis C viruses (HBV and HCV) are life-threatening infections of public health importance due to their association with cirrhosis and hepatocellular carcinoma. Despite HBV being moderately endemic in many low-income countries, there is no routine HBV vaccination among child bearing aged women making them at risk of transmitting infections to the foetus during pregnancy. This study investigated the seroprevalence of antihepatitis B surface antibodies (anti-HBs) and HCV antibodies among pregnant women in Mwanza city to provide data that can be used in devising preventive strategies. Methods. A cross-sectional hospital-based study involving 339 pregnant women was conducted between June and July 2017. Data were collected using structured data collection tool. Detection of anti-HBs was performed using enzyme immunoassay while qualitative rapid immunochromatographic tests were employed to detect HCV antibodies. Data was analyzed by using STATA version 13. Results. The mean age of the study participants was $25.6 \pm 5.8$ years. The prevalence of anti-HBs was $85 / 339$ (25.1\%, 95\% CI: 20.4-29.6) while that of $\mathrm{HCV}$ antibodies was 1/333 (0.3\%, 95\% CI: 0.1-0.4). By univariate logistic regression analysis, increase in age (OR: 1.04, 95\% CI: 1.00-1.09, $\mathrm{P}=0.03$ ), unknown HIV status (OR: 0.3, 95\% CI: 0.11-0.79, P=0.035), and multigravidity (OR: 2.12, 95\% CI: 1.183.8, $\mathrm{P}=0.038$ ) were significantly associated with anti-HBs seropositivity. Conclusion. A significant proportion of pregnant women have anti-HBs while the seroprevalence of $\mathrm{HCV}$ is low among pregnant women in the city of Mwanza. Routine screening of HBV among pregnant women coupled with appropriate management should be emphasized in developing countries. Further studies to determine seroprevalence of $\mathrm{HCV}$ are recommended across the country.

\section{Introduction}

Hepatitis B virus (HBV) and hepatitis $C$ virus are major public health concerns particularly in the sub-Saharan Africa and Asian countries. HBV causes over one million deaths and infections to over 350 million people worldwide. Most of individuals in endemic areas have been infected vertically or during childhood [1]. Similarly, HCV infection is common in many countries worldwide [2]. The World Health Organization (WHO) estimates that $3 \%$ of the world's populations are chronically infected with $\mathrm{HCV}$ with high prevalence reported in the sub-Saharan Africa $[3,4]$. Furthermore, hepatitis A and $\mathrm{E}$ have been documented to have adverse outcomes in pregnancy $[5,6]$. Despite percutaneous inoculation being the well-known mode of transmission of $\mathrm{HCV}$, other previous reports documented other transmission routes such as unprotected sexual intercourse, occupational accidents, and vertical transmission [7].

Infections with HBV and HCV during pregnancy have been associated with high risk of vertical transmission which 
may result in neonatal hepatitis. The highest risk (80\%-90\%) of chronic infection has been observed among neonates with neonatal hepatitis $[8,9]$. Other complications include low birth weight and premature delivery during the acute phase as well as antepartum hemorrhage and preterm delivery during chronic phase [10]. Understanding epidemiology of viral hepatitis during pregnancy is important as the information can be used in devising appropriate control measures.

Different studies in Africa have documented the magnitude of viral hepatitis during pregnancy [11-14] using HBsAg with very few studies assessing other markers such as antiHBs. Despite its importance there is scarcity of data in some of African countries including Tanzania on the magnitude of this infection. In Tanzania, HBV vaccine has been introduced in childhood immunization programme about 5 years ago leaving out the adult population including women of reproductive age. This puts these women at high risk of transmitting the infection during pregnancy. This study was conducted to investigate the prevalence and factors associated with anti-HBs (natural immunity) and $\mathrm{HCV}$ antibodies among pregnant who had no history of HBV vaccination and who were HBsAg negative, the information that may be useful in accelerating efforts to consider incorporating HBV vaccine in other populations including reproductive aged women in endemic areas across the globe.

\section{Materials and Methods}

2.1. Study Design and Study Area. This was a cross-sectional hospital-based study which was conducted from June to July 2017 at Makongoro antenatal clinic in Mwanza city. Mwanza is the second largest city in Tanzania; it is located in the southern shores of Lake Victoria. Makongoro Health Centre is primarily a reproductive and child healthcare facility found in Ilemela district. The clinic serves both Nyamagana and Ilemela districts, with estimated attendance of 60-70 pregnant women per working day.

2.2. Study Population, Inclusion and Exclusion Criteria. The study included all consented pregnant women aged 18 and above at different gestation ages attending Makongoro antenatal clinic during the study period while all women with unknown gestation ages were excluded. The study ecluded all women with history of $\mathrm{HBV}$ vaccination and those who were HBsAg positive.

2.3. Sample Size Estimation and Sampling Technique. Sample size was estimated by using Kish Leslie formula (1965) [15] using the prevalence of $30.2 \%$ [16]. The minimum sample size obtained was 324 pregnant women. However, a total of 339 women were enrolled. Serial sampling based on inclusion and exclusion criteria was used to recruit study participants until the desired sample size was reached.

2.4. Data Collection, Sample Collection, and Laboratory Procedures. Sociodemographic and other relevant information was collected by using pretested structured data collection tool. Venous blood was collected from all consented participants. Blood was aseptically drawn from the cephalic vein using sterile disposable $5 \mathrm{ml}$ syringes and placed in labeled plain vacutainer tubes (Becton, Dickinson and Company, USA). The tubes were kept at upright position at $21-25^{\circ} \mathrm{C}$ and transported to the CUHAS multipurpose laboratory for processing. The sera were separated, placed in labeled cryovials, and stored at $-80^{\circ} \mathrm{C}$ until processing.

Detection of anti-HBs was done using commercial Sandwich Enzyme Immunoassay (EIA) according to the manufacturer's instructions (SIEMENS-Enzygnost Anti-HBs II, Marburg, Germany) while HCV antibodies were detected by using immunochromatographic test as per manufacturer's instructions, ACON one-step HCV test strip (ACON Laboratories, Inc., CA 92121, USA; DIALAB GmbH, Austria).

2.5. Data Management and Analysis. Data were entered into Excel sheet, cleaned, coded, then transferred into STATA version 13.0 for analysis. Proportions were used to summarize categorical variables while mean (standard deviation) and median (interquartile range) were used for continuous variables. Ranksum Mann-Whitney test was used to compare medians. A stepwise logistic regression model was used to test association between outcome variables and associated factors whereby the variables with $\mathrm{P}$ value of $<0.2$ on univariate analysis were subjected to multivariate logistic regression analysis. Odds ratio (OR), $\mathrm{P}$ values, and 95\% confidence interval (CI) were noted. A P value of $<0.05$ was considered as statistically significant.

\section{Results}

3.1. Sociodemographic Characteristics of the Study Participants. The mean age of the study participants was 25.6 \pm 5.8 years while the median gestation age was 23 (IQR: 14-28) weeks. The majority of the participants, 309 (91.5\%), were married. Most of the participants, 218 (64.3\%), had either no formal education or primary education while about half, 186 (54.9\%), of the participants were multigravida (Table 1 ).

3.2. Prevalence of Anti-HBs and HCV Antibodies and Associated Factors among Pregnant Women in Mwanza City. The seroprevalence of anti-HBs was found to be $85(25.07 \%$, 95\% CI: 20.4-29.6). The mean age of anti-HBs seropositive women was significantly higher than that of their counterparts $(26.8 \pm 5.8$ vs. $25.2 \pm 5.7, \mathrm{P}=0.03)$. On univariate logistic regression analysis, increase in age 26.8 \pm 5.8 (OR: 1.04, 95\% CI: 1.00-1.09, $\mathrm{P}=0.03$ ), unknown HIV status (OR: 0.3, 95\% CI: 0.11-0.79, $\mathrm{P}=0.035$ ), and multigravidity (OR: 2.12, 95\% CI: 1.18-3.8, $\mathrm{P}=0.038)$ were significantly associated with anti-HBs seropositivity. By multivariate logistic regression analysis, only unknown HIV status (OR: 0.32, 95\% CI: 0.12$0.86, \mathrm{P}=0.02$ ) was found to be associated with anti-HBs seropositivity (Table 2). Among 333 women who were tested for HCV antibodies, only one $(0.03 \%, 95 \%$ CI: $0.1-0.4)$ was found to be seropositive. None of the factors was found to predict $\mathrm{HCV}$ seropositivity among pregnant women in Mwanza city. 
TABLE 1: Sociodemographic and clinical characteristics of 339 pregnant women attending Makongoro antenatal clinic.

\begin{tabular}{lcc}
\hline Participant characteristics & Number & Percent/Mean/Median \\
\hline Age $\quad 339$ & $25.6 \pm 5.8$ \\
$\quad$ Gestation & 339 & 23 (IQR:14-28) \\
Marital status & & \\
Married & 309 & 91.5 \\
Unmarried & 30 & 8.5 \\
HIV status & & \\
Negative & 281 & 82.8 \\
Positive & 10 & 2.9 \\
Unknown & 48 & 14.2 \\
Education level & & \\
None/primary & 218 & 64.3 \\
Secondary & 95 & 28.2 \\
Tertiary & 26 & 7.7 \\
Gravidity & & \\
Prime gravida & 115 & 34.0 \\
Gravida 2,3,4 & 186 & 54.9 \\
Gravida $>5$ & 38 & 11.2 \\
Anti-HBs Negative & 254 & $74.93 \%$ \\
Anti-HBs Positive & 85 & $25.07 \%$ \\
Titres & & $18.58 \%$ \\
10-100IU/ml & 63 & $2.95 \%$ \\
100-200IU/ml & 10 & $3.54 \%$ \\
$>$ 200IU/ml & 12 & \\
\hline
\end{tabular}

\section{Discussion}

This is the first study to assess the level of anti-HBs and $\mathrm{HCV}$ antibodies among pregnant women population in Mwanza city. The seroprevalence of anti-HBs in the current study was found to be high, which is comparable to a previous study in Kenya that reported anti-HBs prevalence of $30.2 \%[16]$. Nonetheless, the seroprevalence in this study is significantly higher than what has been documented in Brazil and Bangladesh $[17,18]$ with prevalence of $5.7 \%$ and $8.5 \%$, respectively. On the contrary, the seroprevalence in this study is lower than that of a previous study in China which reported seroprevalence of 58.55\% [19]. The possible explanation for such discrepancies could be geographical variation, endemicity status as per WHO [20], and exposure to the risk factors. Tanzania is among the countries under moderate endemicity which might be different from other countries like China where the endemicity is high moderate and Brazil where the endemicity is low (http://www.hpsc.ie/az/EMIToolkit/appendices/app22.pdf).

In this study, only unknown HIV status was found to predict anti-HBs seropositivity. This observation is different from the previous studies which did not show any association between anti-HBs and HIV status[16, 19]. This observation can be explained by the fact that the HIV status reported in this study was based on antenatal records which might not be reliable to some of the participants. There is a possibility that some of the participants who claimed to have unknown status have positive results which might explain such association. Further studies to confirm this association are recommended in this setting.

Another factor which showed association with anti-HBs seropositivity was increased age which is similar to the previous study done in Bangladesh [18]. A possible explanation for such association could be the fact that aged women are more likely to be exposed to the risk factors compared to young women.

Regarding HCV seropositivity, the seroprevalence was found to be $0.03 \%$ which is comparable to other studies done in Sudan and Nigeria that reported prevalence of $0.6 \%$ and $0.4 \%$, respectively [21]. In comparison to other studies done in Egypt, Ivory Coast, and Rwanda which reported prevalence of $6.1 \%, 3.6 \%$, and $2.4 \%[21,22]$, the reported HCV seroprevalence in the current is significantly low. The possible explanation could be different geographical distribution and exposure to risk factors in relation to the study population [21-25]. Another possible explanation could be differences in sensitivity and specificity of the test used in different studies. In the current study, the test used has sensitivity and specificity of $98.9 \%$ and $99.2 \%$ which might be different from other previous studies.

As a limitation, in the present study anti-HBc antibodies were not tested. However, the population studied had not received $\mathrm{HBV}$ vaccination and were $\mathrm{HBsAg}$ negative, so the results obtained are highly indicative of natural anti-HBs antibodies.

\section{Conclusions}

A substantial number of pregnant women have natural anti-HBs while the prevalence of $\mathrm{HCV}$ antibodies is low among pregnant women in the city of Mwanza. However, a significant proportion of these women are susceptible to HBV infection necessitating the need to ensure all reproductive aged women are protected against HBV. Routine screening of HBV infection among pregnant women coupled with appropriate management should be emphasized in resource limited countries where the viral hepatitis is endemic. Further studies on HCV in different cities across the country are highly recommended.

\section{Data Availability}

All data are included in the manuscript. Raw data is available upon request, and the request should be made to the Director of Research and Innovation, Catholic University of Health and Allied Sciences.

\section{Ethical Approval}

The clearance to conduct the study was sought from the joint Catholic University of Health and Allied Sciences/Bugando Medical Centre (CUHAS/BMC) Research Ethics and Review Committee (CREC) with ethical clearance number CREC/344/2017. 
TABLE 2: Factors associated with anti-HBs seropositivity among 339 pregnant women attending Makongoro antenatal clinic.

\begin{tabular}{|c|c|c|c|c|c|c|}
\hline \multirow{3}{*}{ Variable } & \multirow{3}{*}{$\begin{array}{c}\text { Immuno } \\
\text { (Negative) } \\
\text { Median\%(IQR) }\end{array}$} & \multirow{3}{*}{$\begin{array}{c}\text { Immuno } \\
\text { (Positive) } \\
\text { Median\%(IQR) }\end{array}$} & \multicolumn{2}{|c|}{ Univariate analysis } & \multicolumn{2}{|c|}{ Multivariate analysis } \\
\hline & & & OR[95\%CI $]$ & $\mathrm{P}$-value & OR[95\%CI $]$ & P-value \\
\hline & & & & & & \\
\hline Age & $25.2 \pm 5.7$ & $26.8 \pm 5.8$ & $1.04[1.00-1.09]$ & 0.033 & $1.02[0.97-1.08]$ & 0.358 \\
\hline Gestation & 21.5(IQR:14-28) & 24(IQR:16-28) & & & & \\
\hline \multicolumn{7}{|l|}{ Marital S. } \\
\hline Single & $23(76.67)$ & $7(23.33)$ & 1 & & & \\
\hline Married & $231(74.76)$ & $78(25.24)$ & $1.1[0.45-2.68]$ & 0.818 & & \\
\hline \multicolumn{7}{|l|}{$H I V$} \\
\hline Negative & $203(72.24)$ & $78(27.76)$ & 1 & & & \\
\hline Positive & $8(80)$ & $2(20)$ & $0.65[0.13-3.13]$ & 0.592 & $0.47[0.92-2.39]$ & 0.36 \\
\hline Unknown & $43(89.58)$ & $5(10.42)$ & $0.3[0.11-0.79]$ & 0.035 & $0.32[0.12-0.86]$ & 0.02 \\
\hline \multicolumn{7}{|l|}{ Education } \\
\hline None/Pri. & $159(72.94)$ & $59(27.06)$ & 1 & & & \\
\hline Second. & $76(80)$ & $19(20)$ & $0.67[0.37-1.2]$ & 0.405 & & \\
\hline Tertiary & $19(73.08)$ & $7(26.92)$ & $0.99[0.39-2.48]$ & 0.988 & & \\
\hline \multicolumn{7}{|l|}{ Blood splash } \\
\hline No & $241(75.79)$ & $77(24.21)$ & 1 & & & \\
\hline Yes & $13(61.90)$ & $8(38.10)$ & $1.92[0.76-4.8]$ & 0.155 & $1.79[0.68-4.67]$ & 0.23 \\
\hline \multicolumn{7}{|l|}{ Gravidity } \\
\hline Prim gravid & $96(83.48)$ & $19(16.52)$ & 1 & & & \\
\hline Gravid 2,3,4 & $131(70.43)$ & $55(29.57)$ & $2.12[1.18-3.8]$ & 0.038 & $1.75[0.9-3.42]$ & 0.36 \\
\hline Gravid 5 -> & $27(71.05)$ & $11(28.95)$ & $2.05[0.87-4.84]$ & 0.099 & $1.32[0.44-3.91]$ & 0.61 \\
\hline
\end{tabular}

\section{Consent}

A written informed consent was requested from the participants after explaining the study aims before enrolment in the study. For illiterate women, the consent information sheet was read loudly in full and the participants were requested to thumbprint to signify their willingness to participate. Participants were asked to participate voluntarily, and those who were not willing to participate were also given a standard care as per facility protocols.

\section{Conflicts of Interest}

The authors declare that they have no conflicts of interest.

\section{Authors' Contributions}

Mariam M. Mirambo, Elieza Chibwe, and Stephen E. Mshana participated in the design of the study. Caroline A. Minja, Edwin Kajoro, Emmanuel Mkumbo, Elieza Chibwe, Fridolin Mujuni, and Muhsin Juma participated in the data and sample collection. Vitus Silago, Edwin Kajoro, Emmanuel Mkumbo, and Muhsin Juma performed serological tests. Stephen E. Mshana and Mariam M. Mirambo analysed and interpreted the data. Mariam M. Mirambo wrote the first draft of the manuscript. Stephen E. Mshana did a critical review of the manuscript. All authors read and approved the final version of the manuscript.

\section{Acknowledgments}

This study was supported by the Catholic University of Health and Allied Sciences and the Medical Mission Institute, Salvatorstrasse 7, 97067 Wuerzburg, Germany. The authors would like to acknowledge the technical support provided by Makongoro antenatal clinic staff, Bugando Medical Centre laboratory staff, and the Department of Microbiology and Immunology, CUHAS-Bugando.

\section{References}

[1] L. Teresa and M. Wright, "Introduction to chronic hepatitis B infection," The American Journal of Gastroenterology, vol. 101, pp. S1-S6, 2006.

[2] S. Zaltron, A. Spinetti, L. Biasi, C. Baiguera, and F. Castelli, "Chronic HCV infection: epidemiological and clinical relevance," BMC Infectious Diseases, vol. 12, supplement 2, article S2, 2012.

[3] A. Zanetti, "Global surveillance and control of hepatitis C. Report of a WHO Consultation organized in collaboration with the Viral Hepatitis Prevention Board, Antwerp, Belgium," Journal of viral hepatitis, vol. 6, no. 1, pp. 35-47, 1999.

[4] V. Madhava, C. Burgess, and E. Drucker, "Epidemiology of chronic hepatitis $\mathrm{C}$ virus infection in sub-Saharan Africa," The Lancet Infectious Diseases, vol. 2, no. 5, pp. 293-302, 2002.

[5] S. Patra, A. Kumar, S. S. Trivedi, M. Puri, and S. K. Sarin, "Maternal and fetal outcomes in pregnant women with acute hepatitis E virus infection," Annals of Internal Medicine, vol. 147, no. 1, pp. 28-33, 2007. 
[6] S. P. B. Jaiswal, A. K. Jain, G. Naik, N. Soni, and D. S. Chitnis, "Viral hepatitis during pregnancy," International Journal of Gynecology and Obstetrics, vol. 72, no. 2, pp. 103-108, 2001.

[7] M. I. Memon and M. A. Memon, "Hepatitis C: an epidemiological review," Journal of Viral Hepatitis, vol. 9, no. 2, pp. 84-100, 2002.

[8] K. Y. Tse, F. H. Lai, and T. Lao, "The impact of maternal HBsAg carrier status on pregnancy outcomes: A case-control study," Journal of Hepatology, vol. 43, no. 5, pp. 771-775, 2005.

[9] S. E. Dafallah, F. H. El-Agib, and G. O. Bushra, "Maternal mortality in a teaching hospital in Sudan," Saudi Medical Journal, vol. 24, no. 4, pp. 369-372, 2003.

[10] A.-M. Cui, X.-Y. Cheng, J.-G. Shao et al., "Maternal hepatitis $B$ virus carrier status and pregnancy outcomes: A prospective cohort study," BMC Pregnancy and Childbirth, vol. 16, no. 1, article no. 87, 2016.

[11] C. Laurenta, D. Henzel, C. Mulanga-Kabeyaa, G. Maertens, B. Larouzé, and E. Delaporte, "Seroepidemiological survey of hepatitis $\mathrm{C}$ virus among commercial sex workers and pregnant women in Kinshasa, Democratic Republic of Congo," International Journal of Epidemiology, vol. 30, no. 4, pp. 872-877, 2001.

[12] B. Nacro, B. Dao, H. Dahourou et al., "HBs antigen carrier state in pregnant women in Bobo Dioulasso (Burkina Faso)," Dakar Médical, vol. 45, no. 2, pp. 188-190, 2000.

[13] J. Simpore, A. Savadogo, D. Ilboudo et al., "Toxoplasma gondii, $\mathrm{HCV}$, and $\mathrm{HBV}$ seroprevalence and co-infection among HIV-positive and-negative pregnant women in Burkina Faso," Journal of Medical Virology, vol. 78, no. 6, pp. 730-733, 2006.

[14] I. M. Wurie, A. T. Wurie, and S. M. Gevao, "Sero-prevalence of hepatitis B virus among middle to high socio-economic antenatal population in Sierra Leone," West African Journal of Medicine, vol. 24, no. 1, pp. 18-20, 2005.

[15] L. Kish, Survey Sampling, 1965.

[16] F. Okoth, J. Mbuthia, Z. Gatheru et al., "Seroprevalence of hepatitis b markers in pregnant women in Kenya," East African Medical Journal, vol. 83, no. 9, pp. 485-493, 2006.

[17] M. T. Souza, T. L. de Pinho, M. D. Santos et al., "Prevalence of hepatitis B among pregnant women assisted at the public maternity hospitals of São Luís, Maranhão, Brazil," The Brazilian Journal of Infectious Diseases, vol. 16, no. 6, pp. 517-520, 2012.

[18] M. Shamsuzzaman, P. Singhasivanon, J. Kaewkungwal et al., "Hepatitis B among pregnant women attending health care facilities in rural Bangladesh," Southeast Asian Journal of Tropical Medicine and Public Health, vol. 42, no. 6, pp. 1410-1413, 2011.

[19] Y. Ding, Q. Sheng, L. Ma, and X. Dou, "Chronic HBV infection among pregnant women and their infants in Shenyang, China," Virology Journal, vol. 10, no. 1, article no 17, 2013.

[20] Organization WHO, World Health Statistics 2015, World Health Organization, 2015.

[21] M. Mutagoma, H. Balisanga, D. Sebuhoro et al., "Hepatitis $\mathrm{C}$ virus and HIV co-infection among pregnant women in Rwanda," BMC Infectious Diseases, vol. 17, no. 1, article no. 167, 2017.

[22] H. H. Khamis, A. G. Farghaly, H. Z. Shatat, and E. M. el-Ghitany, "Prevalence of hepatitis $\mathrm{C}$ virus infection among pregnant women in a rural district in Egypt," Tropical Doctor, vol. 46, no. 1, pp. 21-27, 2016.

[23] E. Umumararungu, F. Ntaganda, J. Kagira, and N. Maina, "Prevalence of hepatitis C Virus infection and its risk factors among patients attending Rwanda military hospital, Rwanda," BioMed Research International, vol. 2017, Article ID 5841272, 7 pages, 2017.
[24] R. M. Elsheikh, A. A. Daak, M. A. Elsheikh, M. S. Karsany, and I. Adam, "Hepatitis B virus and hepatitis C virus in pregnant Sudanese women," Virology Journal, vol. 4, no. 1, article no 104, 2007.

[25] F. Rouet, M. L. Chaix, A. Inwoley et al., "HBV and HCV prevalence and viraemia in HIV-positive and HIV-negative pregnant women in Abidjan, Côte d'Ivoire: The ANRS 1236 study," Journal of Medical Virology, vol. 74, no. 1, pp. 34-40, 2004. 


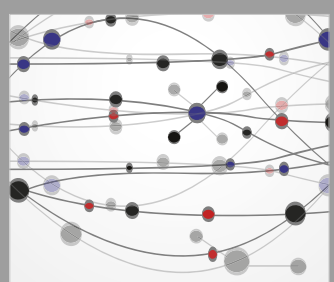

The Scientific World Journal
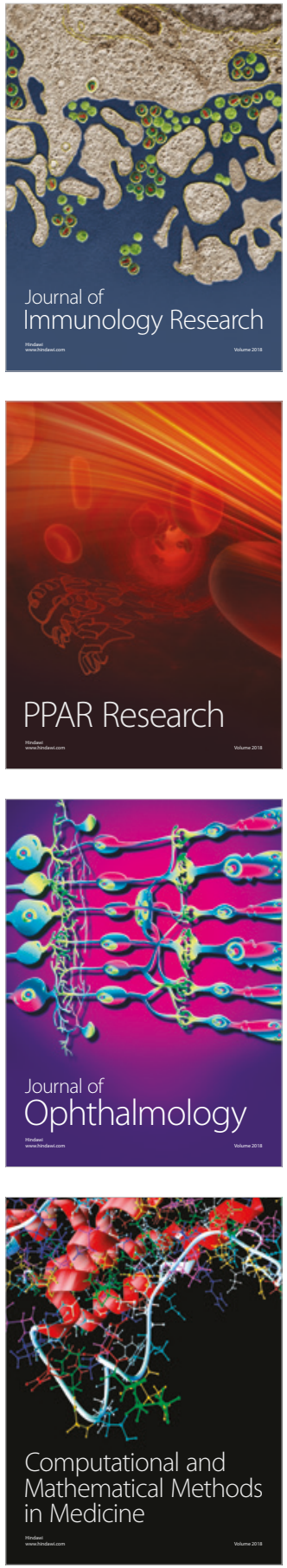

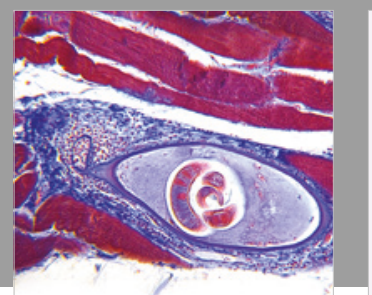

Gastroenterology Research and Practice

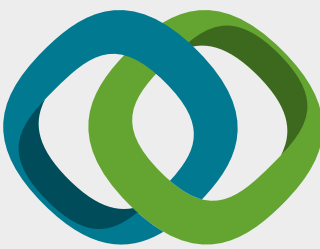

\section{Hindawi}

Submit your manuscripts at

www.hindawi.com
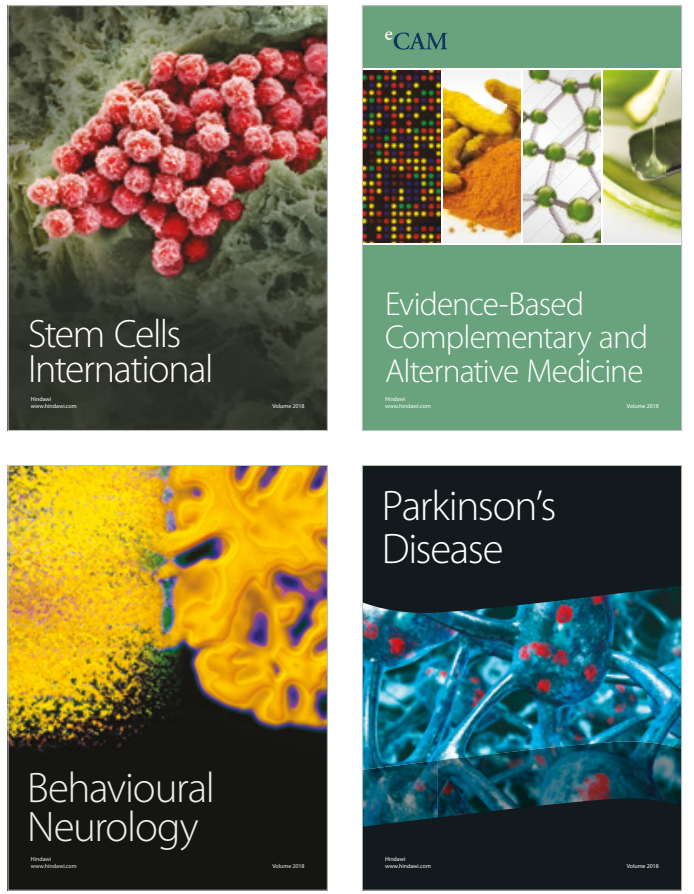

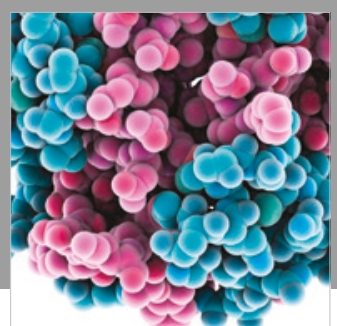

ournal of

Diabetes Research

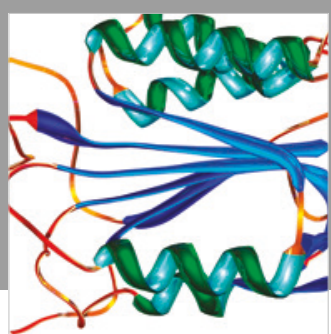

Disease Markers
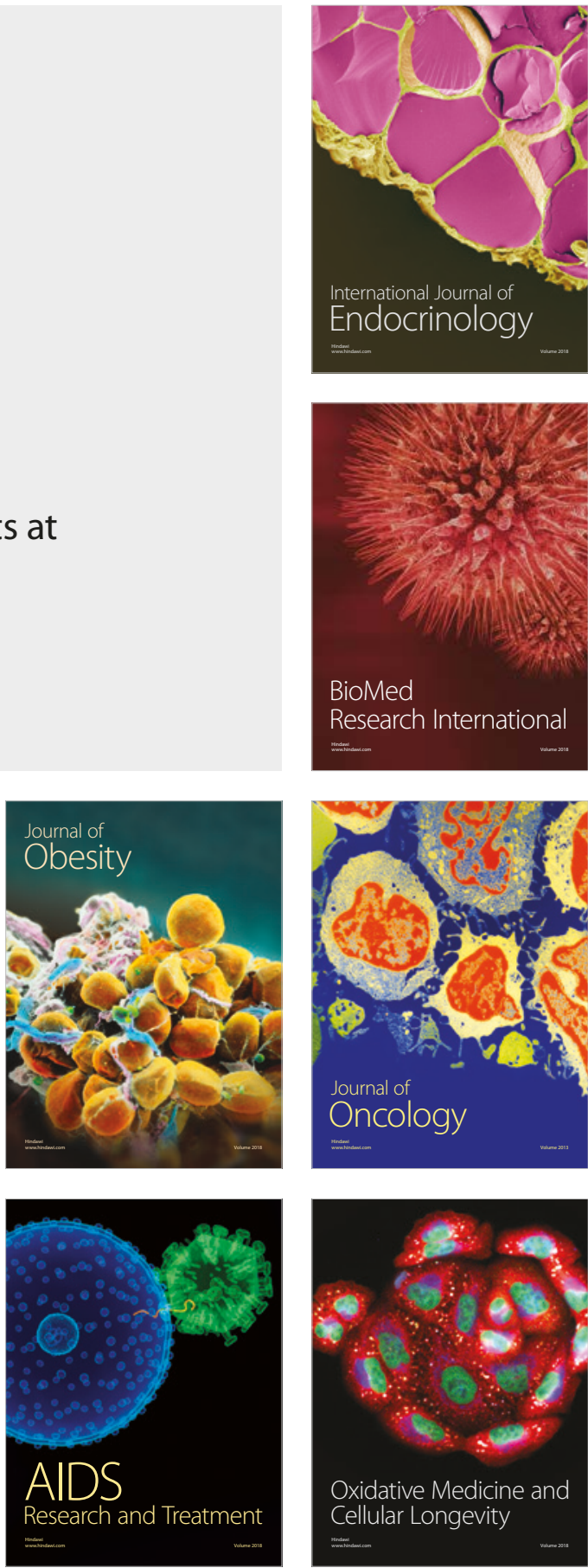